\title{
One-Stage Continuous Melting Transition in Two Dimensions
}

\author{
Julio F. Fernández, ${ }^{1,2, *}$ Juan J. Alonso, ${ }^{2}$ and Jolanta Stankiewicz ${ }^{1}$ \\ ${ }^{1}$ Instituto Venezolano de Investigaciones Científicas, Apartado 21827, Caracas 1020-A, Venezuela \\ ${ }^{2}$ Departamento de Física Aplicada, Universidad de Granada, 18071 Granada, Spain
}

(Received 10 April 1995)

\begin{abstract}
Monte Carlo results are reported for melting of two-dimensional systems of $N$ hard disks for various values of $N$. Runs of up to $10^{8}$ Monte Carlo sweeps give the following equilibrium results: (1) there is a single second-order transition at volume $v_{c}=1.260 \pm 0.005 ;(2)$ the orientational order parameter drops discontinuously to zero at $v=v_{c}$; (3) $\eta_{6}=0.30 \pm 0.05$ at $v=v_{c}$; (4) there is consistency with $\xi=\exp \left(b / u^{1 / 2}\right)$ in the isotropic phase, where $u=v-v_{c}$ and $b \simeq 0.8$. An intermediate phase in which the volume can vary by more than about $1 \%$ is ruled out.
\end{abstract}

PACS numbers: $64.70 . \mathrm{Dv}, 02.70 . \mathrm{Lq}$, 64.70.Fx

Many experiments [1] and Monte Carlo (MC) simulations [2] have been devoted to determine whether melting in two dimensions (2D) unfolds through two continuous transitions as predicted by Kosterlitz, Thouless, Halperin, Nelson, and Young (KTHNY) [3,4]. No consensus about the existence of the "hexatic" phase that would be intermediate between the ordered and the isotropic phases has been established. Its existence has been inferred most often from experiments [5], but not always [6]. On the other hand, most MC simulations [7], but not all [8], suggest that the transition from the ordered to the isotropic phase is of first order. Hysteretic behavior has often been the basis for it.

Simulations have been seriously limited by computer running times. As Zollweg, Chester, and Leung have pointed out, equilibration times for systems of about $10^{4}$ disks can be much longer than $10^{6} \mathrm{MC}$ sweeps [9]. This is illustrated in Figs. 1(a) and 1(b) for 15876 disks. Lee and Strandburg have recently obtained equilibrium results for small systems through MC simulations of up to a few hundred disks for about $10^{7} \mathrm{MC}$ sweeps [7]. Their results are consistent with a first-order phase transition. It is, however, not a firm conclusion because $\xi \ll L$ ( $L$ is a system's linear size and $\xi$ is its correlation length) was far from being fulfilled. Systems of many thousands of particles must be studied since $\xi \gg 10$ in the transition region [7] (see also below). Moreover, very long simulations are needed because $\tau$ rises critically fast with $L$, and exponentially fast with $L$ within a firstorder transition region. Simulations at constant pressure $p$ are even slower: Any attempted change $\Delta v$ must fulfill $\Delta v \sim 1 / N$ lest $\exp (-\beta p N|\Delta v|)$ become forbiddingly small for large $N$.

We report here equilibrium results obtained through MC simulations that are at least an order of magnitude longer than simulations reported previously for systems of comparable sizes. We simulate systems of $N=900$, 3844 , and 15876 hard disks in the $N p T$ ensemble (in which $p$, rather than $v$, is held fixed) [10]. We establish that either the intermediate phase does not exist, or, if it does, its volume range is quite small. Our results are consistent with a single second-order phase transition. First, the crystalline phase is shown to extend up to volume $v_{m} \simeq 1.263$. That follows from the observation that the bond orientation order parameter $\langle\phi\rangle$ (defined below) appears to be size independent for $v<v_{m}$. On the other hand, it turns out that the isotropic phase extends down to volume $v_{i}=1.260 \pm 0.005$. That follows from examining how $\left\langle(\delta \phi)^{2}\right\rangle$ depends on $v$ and on $N$; in addition, $\left\langle(\delta \phi)^{2}\right\rangle$ obeys finite-size scaling in the isotropic phase if we use (1) $\xi_{6} \sim \exp \left(b /\left|v-v_{i}\right|^{1 / 2}\right)$, following KTHNY [3], (2) $v_{i}=1.260 \pm 0.005, b=0.8 \pm 0.1$, and (3) the algebraic decay critical index value $\eta_{6}=$ $0.30 \pm 0.05$ at $v=v_{i}$. Our data suggest that $\langle\phi\rangle$ drops
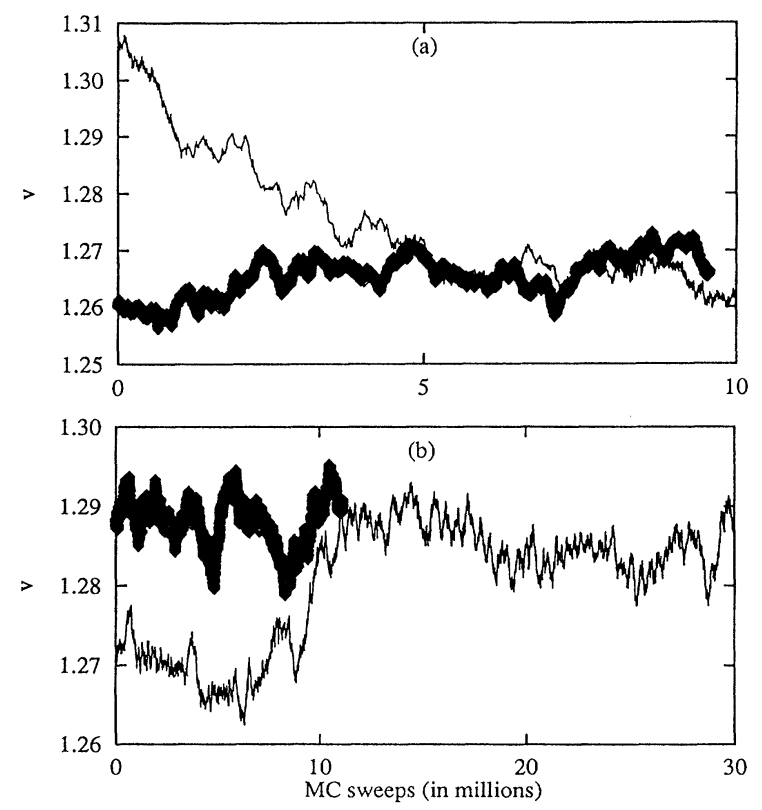

FIG. 1. (a) Volume vs MC sweeps for 15876 disks, for $p=7.90$, for two different initial configurations, obtained from previously expanding (compressing) a dense (dilute) ordered (disordered) state. (b) Same as in (a) but for $p=7.875$. 
off discontinuously to zero (from a value of about 0.74 ) at $v=1.260$. Volume fluctuations that seem consistent with a first-order phase transition for smaller systems [7] decrease as system sizes increase; they clearly show that if the phase transition should ultimately turn out to be of first order the corresponding volume change would have to be smaller than about 0.013 (which is approximately $\frac{1}{4}$ of the value often obtained from MC simulations).

An outline of our simulations follows [11]. Runs vary in length with $N$ and with $p$. In addition to many shorter runs, at least seven runs were performed in the $(40-100) \times 10^{6} \mathrm{MC}$ sweeps range for $N=900,5$ runs in the $(20-50) \times 10^{6} \mathrm{MC}$ sweeps range for $N=3844$, and 8 runs in the $(10-35) \times 10^{6} \mathrm{MC}$ sweeps range for $N=15876$. Data points for our largest systems were obtained from pairs of runs - one of them coming from a disordered state and the other one originating from a crystalline configuration. Averages were performed only after convergence had been achieved. We use "hardcrystalline wall" (HCW) boundary conditions. Consider a rectangular box of $2^{n} \times 2^{n}$ sites on a triangular lattice, and let all sites on the boundary be occupied by disks. They make up the "crystalline" walls. Their positions always remain fixed. $N=\left(2^{n}-2\right)^{2}$ disks inside the box are the system's "particles." All $2^{2 n}$ disks are of equal size and remain so as their radii vary in unison (simulating volume variations of the system). For $v \simeq 4 \times a_{0}$, where $a_{0}$ is the closest packing volume (an area, really), escape through gaps between fixed disks would be possible if there were no additional constraints, such as periodic boundary conditions (PBC). For $v>4 a_{0}$ these conditions may be thought of as PBC plus a symmetry breaking array of fixed scatterers on the boundaries. Here $\langle v\rangle<1.5 v_{0}$ and $\left\langle(v-\langle v\rangle)^{2}\right\rangle^{1 / 2}<0.014$ (see below). Consequently, $v$ never even came close to $\frac{1}{2}$ of $4 a_{0}$ in our simulations. The $\mathrm{HCW}$ conditions behaved therefore as impenetrable walls throughout. We use HCW's in order to ease nucleation of crystal growth, much as crystals are often grown from the melt on a substrate in the laboratory. Furthermore, HCW's do not allow the formation of domain strips across toroidal surfaces that sometimes hinder equilibration of systems with PBC. Systems of $10^{3}$ disks, for instance, turn out to equilibrate at least 10 times faster for $\mathrm{HCW}$ than for PBC. Clearly, results for finite system with HCW and with PBC must differ some, just as fixed $v$ and fixed $p$ ensemble results differ. However, we draw conclusions for the thermodynamic limit (through finite-size scaling, for instance), and one expects those conclusions to be boundary independent, much as one does in analogous experimental work. $p$ is given in units of $k T / a_{0}$ throughout, where $k$ is Boltzmann's constant, and $T$ is the temperature. All "volumes" are given in units of $a_{0}$. In order to compute errors, each run was divided into five intervals; the corresponding mean values were then used to calculate standard deviations, which are given as errors.
"Time" evolutions of $v$ are shown in Figs. 1(a) and 1(b) for systems of 15876 disks for $p=7.90$ and 7.875 , respectively. The purpose of these figures is to illustrate that there is no hysteresis in our results. The two initial configurations for the larger values of $v$ (which are disordered) were previously compressed from a dilute system; the two initial configurations for smaller $v$ (which are somewhat ordered) were previously evolved at higher pressures starting from a completely ordered system. The final state of Fig. 1(a) [1(b)] is disordered (ordered). All our equilibrium averages for very slow evolving systems (i.e., for $N=15876$ and $7.80 \leq p \leq$ 7.90) were obtained from pairs of runs such as these, after discarding the initial equilibration portions.

It is useful to define a bond orientation order parameter $\phi$ as Halperin and Nelson have [3]. Let $\theta_{n}^{m}$ be the angle between the "bond" linking particles $n$ and $m$ and some arbitrary reference line, and assume a triangular crystal lattice. Then $\left|a_{n}\right| \exp \left(i 6 a_{n}\right) \equiv z_{n}^{-1} \sum_{m} \exp \left(6 i \theta_{n}^{m}\right)$, where $z_{n}$ is the number of suitably defined nearest neighbors [12] of the $n$th particle, defines $\left|a_{n}\right|$ and angle $\alpha_{n} . \alpha_{n}$ is a local crystalline orientation angle. Let

$$
\phi \equiv \tilde{N}^{-1} \sum_{n}\left|a_{n}\right| e^{i 6 \alpha_{n}},
$$

where the sum is only over whatever particles are within a small inner box (that holds only $\tilde{N}$ particles, and $\langle\tilde{N}\rangle=$ $N / 16$ ) within the hard wall box that holds the whole system of $N$ particles.

Pictures and Bragg scatteringlike graphs are shown in Fig. 2 for each of the four final states of Figs. 1(a) and 1(b). Angle $\alpha$ [defined above Eq. (1)] is shown on columns 1 and 3 as a function of position. Red stands for $\alpha \simeq 0^{\circ}$, and so on up to indigo for $\pm 30^{\circ}$. Boundary conditions are biased for $\alpha \simeq 0$ near the walls. The blueish graphs show the structure factor $S(\mathbf{k})$ for inner subsystems of $N / 16$ particles. The left half (right) of the figure is for the final states of Fig. 1(a) [Fig. 1(b)], for which $p=7.90$ $(p=7.875$ ). Everything on the upper (lower) row is for final configurations obtained from initially ordered (disordered) configurations at lower (higher) volumes. The final configurations show no noticeable dependence on initial conditions. The slight anisotropy shown by the Bragg-like graphs on the far right are to be expected, since $\xi_{6} \sim 100$ therein (using $b=0.8, v_{i}=1.26$, and $v=1.29$ ), which is comparable to $L=128$.

Quantity $\langle\phi\rangle$ is shown versus $\langle\boldsymbol{v}\rangle$ in Fig. 3(a) for three values of $N$. There is no appreciable size dependence for $v<1.263$ approximately. Hence, we (temporarily) set $v_{m} \simeq 1.263$. However, it is not immediately obvious from this graph either how or where $\langle\phi\rangle$ will vanish for macroscopic systems.

We now examine quantity $\left\langle(\delta \phi)^{2}\right\rangle$, where $\delta \phi=\phi-$ $\langle\phi\rangle$, in order to determine the extent of the isotropic phase. $\left\langle(\delta \phi)^{2}\right\rangle$ is expected to rise as $v$ decreases, until the rise is arrested as $\xi_{6} \rightarrow L$. More precisely, it follows from 
(a)
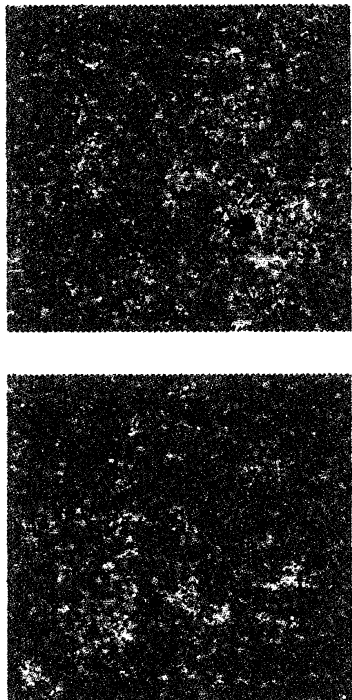

(e) (b)
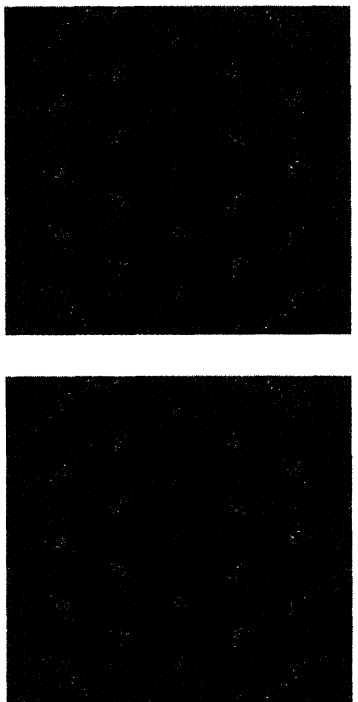

(f) (c)
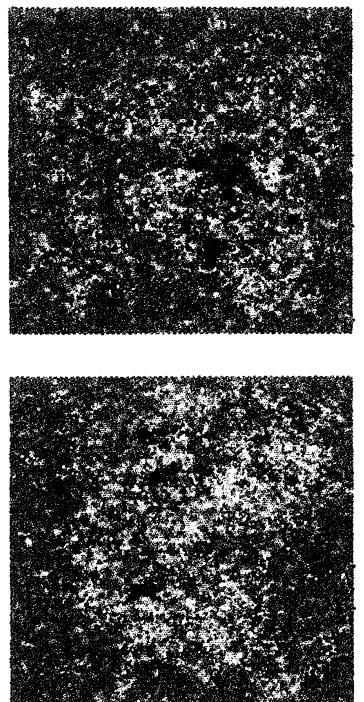

(g) (d)
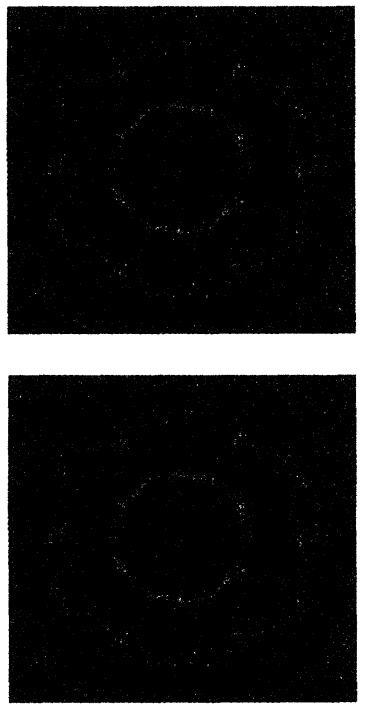

(h)

FIG. 2(color). Pictures on columns 1 and 3 show angle $\alpha$ as a function of position (i.e., Voronoi cell). Red stands for $\alpha \simeq 0^{\circ}$, and so on, up to indigo for $\alpha \simeq \pm 30^{\circ}$. The structure factor $S(\mathbf{k})$ is shown on columns 2 and 4 . Pictures (a) and (b) [(e) and (f)] are for the final states of the runs shown in Fig. 1(a) that started from an ordered [disordered] state. Pictures (c) and (d) [(g) and (h)] are for the final states of the runs shown in Fig. 1(b) that started from an ordered [disordered] state. The final state volumes for a-b, c-d, e-f, and g-h are 1.266, 1.265, 1.285, and 1.290, respectively.

the finite-size scaling expression $\left\langle(\delta \phi)^{2}\right\rangle \sim L^{-\eta_{6}} f\left(\xi_{6} / L\right)$, that maxima of $\left\langle(\delta \phi)^{2}\right\rangle$ correspond to values of $\xi_{6} / L$ that are independent of $N$. Figure 3(b) shows $\left\langle(\delta \phi)^{2}\right\rangle$ vs $v$ for various system sizes. There is a clear-cut trend for the position of maxima to go to $\langle v\rangle \approx 1.265$ as $N \rightarrow \infty$. The inset in Fig. 3(b) shows $L^{\eta_{6}}\left\langle(\delta \phi)^{2}\right\rangle$ vs $b^{-1} \ln \left(\xi_{6} / L\right)$ in the isotropic phase; it is obtained using $\xi_{6}=\exp \left(b / u^{1 / 2}\right)$, where $u=\left|v-v_{i}\right|$, with $v_{i}=1.260, b=0.77$, and $\eta_{6}=0.3$. Scaling becomes significantly worse for $\mid v_{i}-$ $1.260 \mid>0.005$. That, plus the corresponding variations of $b$ and $\eta_{6}$, gives $v_{i}=1.260 \pm 0.005, b=0.8 \pm 0.1$, and $\eta_{6}=0.30 \pm 0.05$. Since $v_{i} \geq v_{m}$ by definition, and we have obtained $v_{m} \simeq 1.263$, we conclude that $v_{i}=$ $v_{m}=1.260$, within statistical errors (which we estimate to be about 0.005). Furthermore, since $\langle\phi\rangle \simeq 0.74$ at $v=$ $v_{m}=1.260$ [by inspection of Fig. 3(a)] and $v_{i}=v_{m}$, we conclude that $\langle\phi\rangle$ drops discontinuously to 0 at the critical point.

Further numerical evidence that is inconsistent with a first-order phase transition follows. $p$ vs $\langle v\rangle$ datapoints are shown in Fig. 4(a). Using $\partial v / \partial p=-N\left\langle(\delta v)^{2}\right\rangle$, we obtain $\left\langle(\delta v)^{2}\right\rangle$ taking derivatives of cubic spline fits to $p(v) .\left\langle(\delta v)^{2}\right\rangle$ is shown in Fig. 4(b). Data points obtained from fluctuations are also shown. Clearly it can safely be stated that $\left\langle\delta v^{2}\right\rangle<4 \times 10^{-5}$ in the $N \rightarrow \infty$ limit. On the other hand, for first-order phase transitions, the maximum value of $\left\langle(\delta \boldsymbol{v})^{2}\right\rangle \rightarrow\left(v_{2}-v_{1}\right)^{2} / 4$ as $N \rightarrow \infty$, where $v_{1}$ and $v_{2}$ are the two hypothetical phase volumes.
Hence, $\left|v_{2}-v_{1}\right|<0.013$, which is to be contrasted with the value $\left|v_{2}-v_{1}\right| \simeq 0.05$ that has often been inferred from MC simulations [2,7].

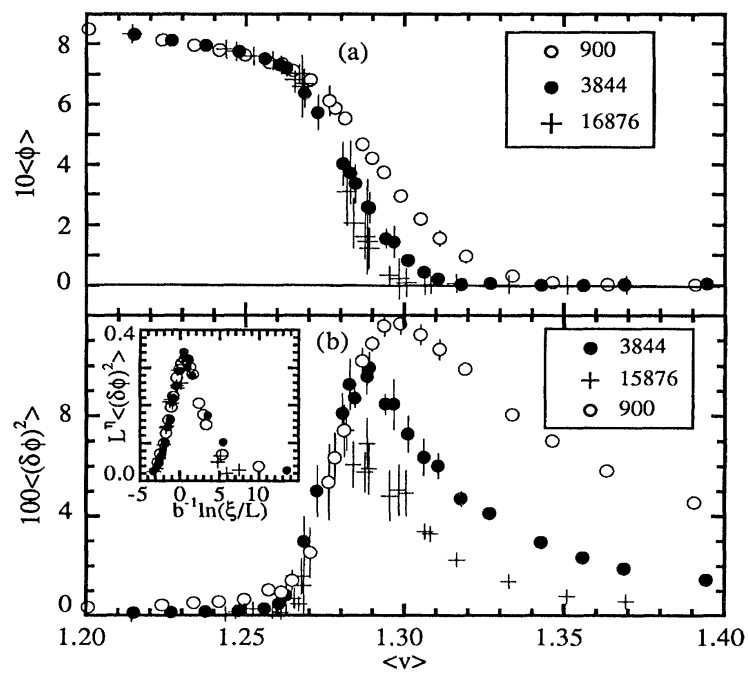

FIG. 3. (a) Quantity $\langle\phi\rangle$ vs $\langle v\rangle$ for the shown values of $N$ hard disks. (b) $\left\langle(\delta \phi)^{2}\right\rangle$ vs $\langle v\rangle$ for various values [same as in Fig. 1(a)] of $N$ hard disks; $\delta \phi \equiv \phi-\langle\phi\rangle . \quad L^{\eta_{6}}\left\langle(\delta \phi)^{2}\right\rangle$ vs $b^{-1} \ln \left(\xi_{6} / L\right)$ is shown in the inset; $\eta_{6}=0.3, b=0.77$, $\xi_{6}=\exp \left(b / u^{1 / 2}\right), u=v-v_{i}$, and $v_{i}=1.260$. 


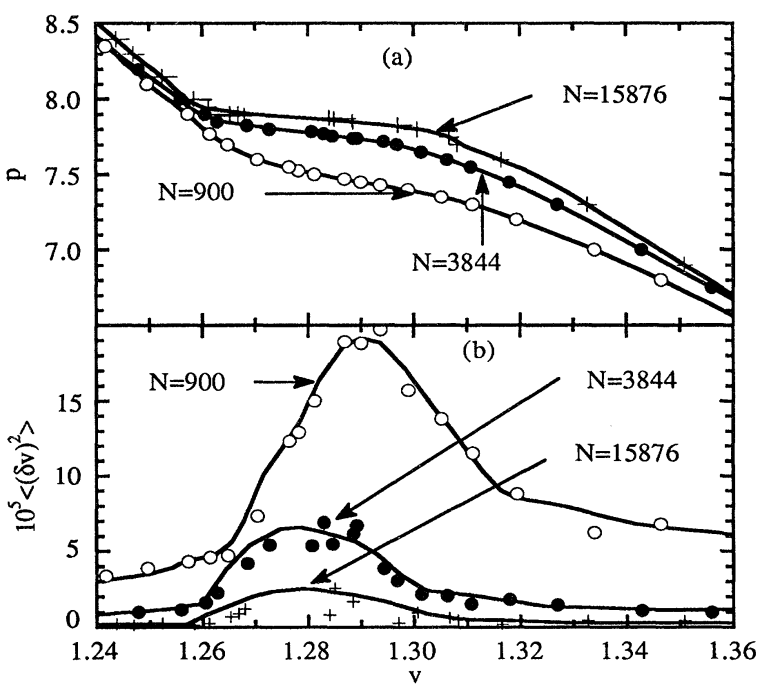

FIG. 4. (a) Data points for $p$ (in units of $k T / a_{0}$, where $a_{0}$ is the close-packed area per disk) vs $v$ for systems of $N$ disks. Error bars are horizontal. They hardly show because of their small size. (b) $\left\langle(\delta v)^{2}\right\rangle$ vs $\langle v\rangle$ for the same systems as in (a). Full lines are for $N^{-1} \partial v / \partial p$, obtained from cubic spline fits to $p(v)$ curves in (a). The points shown are obtained from fluctuations.

How is this to be reconciled with the conclusion most often drawn from MC simulations, that the transition is of first order? To be brief, we shall discuss only the results of Lee and Strandburg [7], which are most recent and are quite clearly stated. The thrust of their argument is that $\Delta G \propto L$ for $N \sim 100$, where $\Delta G$ is the Gibbs free energy barrier for nucleation of another phase at coexistence. That is as expected for a first-order phase transition in 2D. We have also simulated small systems $(N=64$ and 256) with PBC [11]. Agreement with Ref. [7] is obtained. However, our results for larger systems with PBC (from many MC runs of $10^{8} \mathrm{MC}$ sweeps for 1024 disks) do not follow the trend shown by smaller systems. We obtain $\Delta G=0.55 \mathrm{kT}$ for both $N=256$ and $N=1024$, within an error of $10 \%$. That is in line with $\Delta G / L \rightarrow 0$ as $N \rightarrow \infty$. Furthermore, $v_{2}-v_{1}$ is $20 \%$ smaller for $N=$ 1024 than for $N=256$, in accordance with our discussion above about how $\left\langle(\delta v)^{2}\right\rangle$ decreases as $N$ increases.

Our conclusions are given in the third introductory paragraph. Our computer work was performed on many workstations in well over a year's time.
We thank Dr. J. Rivero for much help, Junta de Andalucia, DGCIT (Grant No. PB91-0709) for financial support, and (J. J. A.) Laboratory PMMH (ESPCI, Paris) for its hospitality.

*Present address: ICMA-CSIC, Universidad de Zaragoza, 50009 Zaragoza, Spain.

Electronic address: JULIOF@CC.UNIZAR.ES

[1] For a review and further references to older work, see R. J. Birgeneau and P. M. Horn, Science 232, 329 (1986).

[2] For reviews, see D. Levesque and J.J. Weiss, Topics Appl. Phys. 71, 121 (1992); K. J. Strandburg, Rev. Mod. Phys. 60, 160 (1988). For molecular dynamics simulations, see W. Vermöhlen and N. Ito, Int. J. Mol. Phys. C 5, 1021 (1994).

[3] J. M. Kosterlitz and D. J. Thouless, J. Phys. C 6, 1181 (1973); B. I. Halperin and D. R. Nelson, Phys. Rev. Lett. 41, 121 (1978); D. R. Nelson and B. I. Halperin, Phys. Rev. B 19, 2457 (1979); 21, 5312 (1980); A.P. Young, Phys. Rev. B 19, 1855 (1979).

[4] For alternate theories, see S. T. Chui, Phys. Rev. B 28, 178 (1983).

[5] R. E. Kusner, J. A. Mann, J. Kerins, and A. J. Dahm, Phys. Rev. Lett. 73, 3113 (1994), and references therein.

[6] R. Seshadri and R. M. Westervelt, Phys. Rev. Lett. 66, 774 (1991); Phys. Rev. B 46, 5142 (1992); Phys. Rev. Lett. 70, 234 (1993), and references therein.

[7] J. Lee and K. J. Strandburg, Phys. Rev. B 46, 11190 (1992); J. A. Zollweg and G. V. Chester, Phys. Rev. B 46, 11186 (1992); for older work, see J. Tobochnik and G. V. Chester, Phys. Rev. B 25, 6778 (1982); A. F. Baker, C. Bruin, and H.J. Hilshorst, Phys. Rev. Lett. 52, 449 (1984).

[8] C. Udink and J. van der Elsken, Phys. Rev. B 35, 279 (1987).

[9] J. A. Zollweg, G. V. Chester, and P. W. Leung, Phys. Rev. B 39, 9518 (1989); see also J. J. Morales, E. Velasco, and S. Toxvaerd, Phys. Rev. E 50, 2844 (1994).

[10] W. W. Wood, in Physics of Simple Liquids, edited by H.N. V. Temperley, J.S. Rowlinson, and G.S. Rushbrooke (John Wiley and Sons, Inc., New York, 1968), pp. 114-230; see also K. Strandburg [7].

[11] Details about our MC algorithm, computer runs, and results (including results for $\mathrm{PBC}$ ) will be published elsewhere.

[12] Voronoi cells are defined in M. Senechal, Science 260, 1170 (1993); A. Gervais, J. P. Troadec, and J. Lemaitre, J. Phys. A 25, 6169 (1992). 
(a)
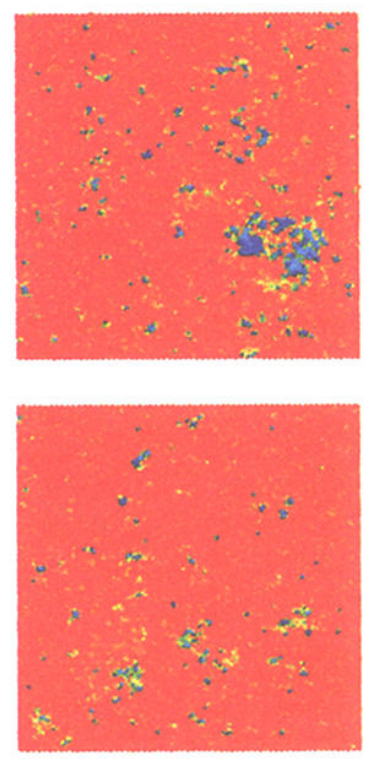

(e) (b)
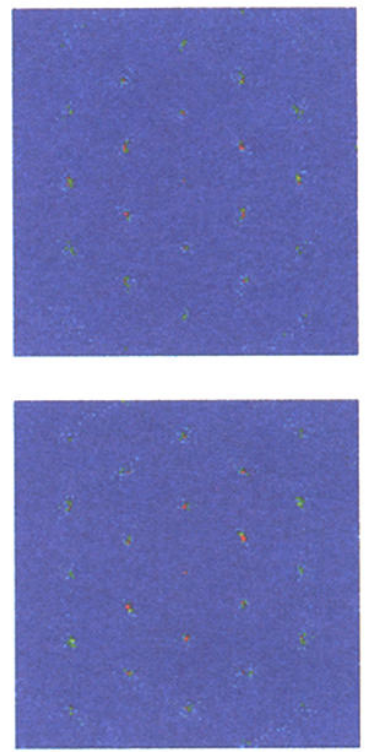

(f) (c)

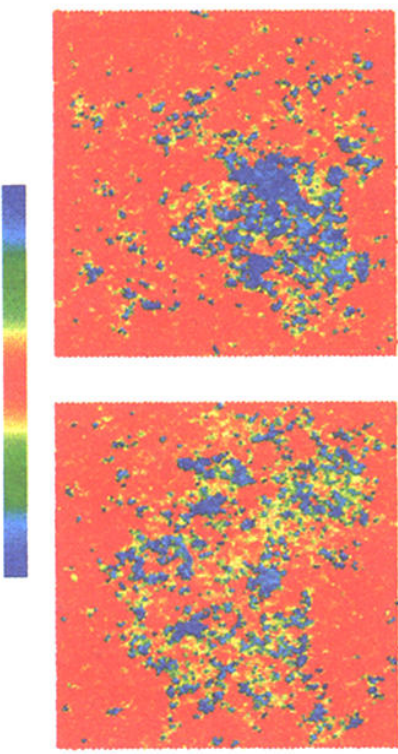

(g) (d)
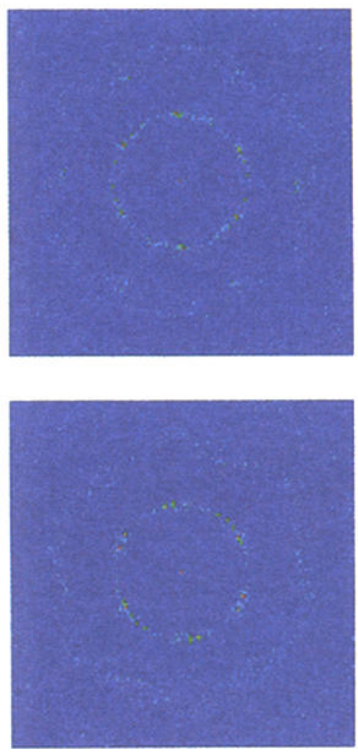

(h)

FIG. 2(color). Pictures on columns 1 and 3 show angle $\alpha$ as a function of position (i.e., Voronoi cell). Red stands for $\alpha \simeq 0^{\circ}$, and so on, up to indigo for $\alpha \simeq \pm 30^{\circ}$. The structure factor $S(\mathbf{k})$ is shown on columns 2 and 4. Pictures (a) and (b) [(e) and (f)] are for the final states of the runs shown in Fig. 1(a) that started from an ordered [disordered] state. Pictures (c) and (d) [(g) and (h)] are for the final states of the runs shown in Fig. 1(b) that started from an ordered [disordered] state. The final state volumes for a-b, c-d, e-f, and g-h are 1.266, 1.265, 1.285, and 1.290, respectively. 